\title{
The link between obesity and psoriatic arthritis
}

\author{
Juan D Cañete, ${ }^{1}$ Philip Mease ${ }^{2}$
}

Psoriatic arthritis (PsA) is classified as a spondyloarthropathy and is characterised by synovitis, enthesitis, dactylitis and spondylitis, usually following the development of cutaneous and nail psoriasis. ${ }^{1}$ PsA patients have a higher prevalence of some comorbidities (obesity, metabolic syndrome, depression and premature cardiovascular disease) than patients with psoriasis (Pso) without arthritis. ${ }^{2}$ Increased adiposity and weight gain have been associated with the risk of psoriasis in a prospective study, ${ }^{3}$ and because $10-30 \%$ of Pso patients develop PsA, it is increasingly important to determine whether obesity is solely a comorbid condition in PsA or a risk factor.

A case-control study in patients with Pso found that patient-reported body mass index (BMI) at age 18 years, but not current BMI, was associated with the development of PsA. ${ }^{4}$ However, the study had some important limitations, including the case definition of PsA, difficulties in establishing a temporal relationship between the factors evaluated, and a lack of validated data on the accuracy of patient recall of BMI at 18 years of age..$^{5}$

This issue of the journal includes two large prospective studies that suggest obesity is a risk factor for PsA. ${ }^{7}$ The cohort study by Love et al, which was conducted using an electronic database of medical records representative of the general UK population and with a 15-year time horizon, found the incidence rates of PsA increased in tandem with BMI, both in the 75395 people with psoriasis and in the general population (almost 2 million), independent of age, sex, trauma, smoking and alcohol intake. ${ }^{6}$ $\mathrm{Li}$ et al analysed information on BMI, weight change and measures of central obesity in participants in the US Nurse Health Study II (89 049 women) with a

\footnotetext{
${ }^{1}$ Arthritis Unit, Department of Rheumatology, Hospital Clinic and IDIBAPS, Barcelona, Spain

${ }^{2}$ Seattle Rheumatology Associates, Swedish Medical Center, University of Washington, Seattle, Washington, USA
}

Correspondence to Dr Juan D Cañete, Arthritis Unit, Department of Rheumatology, Hospital Clinic, Barcelona, Spain; jcanete@clinic.ub.es 14-year time horizon. They found that BMI was monotonically associated with an increased risk of incident PsA. Moreover, there was a graded positive association between weight change from 18 years of age onwards and measures of central obesity, and the risk of PsA. A similar association was found in participants developing psoriasis during the follow-up. The authors concluded that obesity is an independent risk factor for PsA. ${ }^{7}$

These studies offer valuable new information on the link between obesity and PsA and provide a potential opportunity to reduce the occurrence of PsA by encouraging a reduction in weight, a modifiable risk factor. However, some methodological concerns should be taken into account. Firstly, the case definition of PsA in the study by Love et al is dependent on the diagnosis of the primary care physician, as neither validated screening questionnaires nor confirmation by a rheumatologist were used, and therefore the sample could have included patients with osteoarthritis or other rheumatic diseases. Li et al used case ascertainment of clinically diagnosed PsA according to the Psoriatic Arthritis Screening and Evaluation (PASE) questionnaire, a validated screening tool, among self-reported psoriatic patients. It is not clear how the authors treated the variables over time. For example, study of BMI over time provides more accurate information on its effect in PsA; since BMI was not measured during the same period in all study participants, it would be advantageous to know not only whether a patient had a high BMI, but also for how long they had it. A further concern is whether obesity is a true independent risk factor or not. The authors state that it is an independent factor because, in the multiple regression analysis, after controlling for other variables, obesity remained statistically significant. However, some caution is necessary, because, in studies with large sample sizes, small differences may result in significant differences.

Nevertheless, the similar results obtained in these two studies carried out in very different populations and the dosedependent association between adiposity and the development of PsA found by both reinforce the conclusion that obesity is a risk factor for PsA.

What is the physiopathological basis of the link between obesity and PsA? Clearly, more insight on the differential genetics of Pso and PsA and a better definition of the PsA phenotype associated with obesity is required. Are there differences in the patterns of PsA associated or not with obesity? While awaiting further studies that may clarify this issue, it may be timely to review the evidence linking obesity and inflammation.

Obesity leads to increased inflammatory cytokines (IL-6, TNF $\alpha$ ) and changes in related molecules such as leptin and adiponectin, which may contribute to the development of multiple disturbances in predisposed individuals. However, in most cases the relationship appears to be bi-directional, as comorbidities seem to increase and perpetuate the proinflammatory status associated with adiposity. ${ }^{8}$ Therefore, the 'obesity of psoriasis' is thought to be a key link to the increased risk of diabetes, metabolic syndrome and cardiovascular disease, but the proinflammatory molecules produced in these conditions also increase susceptibility to psoriasis and the severity of established psoriasis. $^{9}$

Adipose tissue is composed mainly of adipocytes and the stromo-vascular fraction, which includes macrophages. Fat accumulation results in the upregulation of cell adhesion molecules and chemokines such as monocyte-chemoattractan protein-1 (MCP-1). MCP-1 might contribute to increased production of adiposetissue-resident macrophages, which increase in proportion to BMI and could contribute to the inflammatory state. ${ }^{10}$ Endoplasmic reticulum (ER) stress activation has been demonstrated in adipocytes due to the need to synthesise proteins for the formation of lipid droplets, the production of enzymes and the conversion of energy to triglycerides at times of overnutrition. As occurs in spondyloarthritis, ${ }^{11}$ ER stress induces the cellular inflammatory cascade through the c-Jun $\mathrm{N}$-terminal kinase (JNK) pathway, and JNK has been shown to be upregulated in the adipose tissue of obese individuals, leading to immuno-inflammatory responses. ${ }^{12}$ Angiogenesis, with an increased number of synovial vessels with a characteristic morphology, seems to characterise PsA from its early stages. ${ }^{13}$ It has been shown that adipose tissue in 
severely obese patients produces proangiogenic factors, including vascular endothelial growth factor (VEGF) and placental growth factor and, in these patients, BMI correlated with vascular density in subcutaneous white adipose tissue. However, after effective weightlowering treatment, these abnormalities are reversed. ${ }^{14}$ Taken together, these data suggest that obesity has the potentiality to initiate many of the known immuneinflammatory pathways underlying the pathogenesis of PsA.

There is a possible epidemiological link between PsA and joint trauma: normal entheses show evidence of microscopic damage and associated inflammatory changes. Therefore, it has been suggested that persons at risk of PsA could have an aberrant response to tissue microdamage in diverse sites. ${ }^{15}$

Could obesity-related systemic inflammation become localised at the joints and/or entheses due to the biomechanical or tissue-specific factors that have already been proposed as predictors of arthritis in patients with psoriasis? ${ }^{16}$ As stated, it is not yet clear whether a specific pattern of PsA is associated with obesity. However, the fact that no association has been demonstrated between obesity and seropositive rheumatoid arthritis could reflect the more autoinflammatory nature of PsA and the relevance of tissueassociated factors in this disease. ${ }^{17}$

Beyond their physiopathological correlates, the findings on obesity and PsA are clinically relevant and will spur interest in research aimed at understanding the immune/inflammatory mechanisms linking obesity with PsA and improved management of patients with PsA. Therefore, clinically, it would be of utility to confirm whether preventing or treating obesity may serve as a preventive and/or concomitant therapeutic measure for Pso and PsA. Understanding and implementation of these measures could reduce the severe comorbidity associated with psoriatic disease. $^{18}$

\section{Competing interests None.}

Provenance and peer review Commissioned; externally peer reviewed.

Accepted 30 May 2012

Ann Rheum Dis 2012;71:1265-1266.

doi:10.1136/annrheumdis-2012-201632

\section{REFERENCES}

1. Mease P. Psoriatic arthritis: update on pathophysiology, assessment and management. Ann Rheum Dis 2011;70(Suppl 1):i77-84.

2. Husted JA, Thavaneswaran A, Chandran V, et al. Cardiovascular and other comorbidities in patients with psoriatic arthritis: a comparison with patients with psoriasis. Arthritis Care Res (Hoboken) 2011; 63:1729-35.

3. Setty AR, Curhan G, Choi HK. Obesity, waist circumference, weight change, and the risk of psoriasis in women: Nurses' Health Study II. Arch Intern Med 2007;167:1670-5.

4. Soltani-Arabshahi R, Wong B, Fen BJ, et al. Obesity in early adulthood as a risk factor for psoriatic arthritis. Arch Dermatol 2010;146:721-6.

5. Ogdie A, Gelfand JM. Identification of risk factors for psoriatic arthritis: scientific opportunity meets clinical need. Arch Dermatol 2010;146:785-8.
6. Love TJ, Zhu Y, Zhang Y, et al. Obesity and the risk of psoriatic arthritis: a population-based study. Ann Rheum Dis 2012;71:1273-7.

7. Li W, Han J, Qureshi AA. Obesity and risk of incident psoriasis arthritis in US women. Ann Rheum Dis 2012. In press.

8. Gualillo $\mathbf{0}$. Mediators of inflammation in obesity and its comorbidities. Mediators Inflamm 2010. doi:10.1155/2010/239126

9. Davidovici BB, Sattar N, Jörg PC, et al. Psoriasis and systemic inflammatory diseases: potential mechanistic links between skin disease and co-morbid conditions. J Invest Dematol 2010; 138:1785-96

10. $\mathbf{X u ~ H , ~ B a r n e s ~ G T , ~ Y a n g ~ Q , ~ e t ~ a l . ~ C h r o n i c ~ i n f l a m m a t i o n ~}$ in fat plays a crucial role in the development of obesity-related insulin resistance. J Clin Invest 2003;112:1821-30.

11. Colbert RA, DeLay ML, Klenk El, et al. From HLA-B27 to spondyloarthritis: a journey through the ER. Immunol Rev 2010;233:181-202.

12. Sharma NK, Das SK, Mondal AK, et al. Endoplasmic reticulum stress markers are associated with obesity in nondiabetic subjects. J Clin Endocrinol Metabol 2008;93:4532-41.

13. Fearon U, Griosios K, Fraser A, et al. Angiopoietins, growth factors, and vascular morphology in early arthritis. J Rheumatol 2003;30:260-8.

14. Lemoine AY, Ledoux S, Quéguiner I, et al. Link between adipose tissue angiogenesis and fat accumulation in severely obese subjects. J Clin Endocrinol Metab 2012;97:E775-80.

15. Ash ZR, Tinazzi I, Gallego CC, et al. Psoriasis patients with nail disease have a greater magnitude of underlying systemic subclinical enthesopathy than those with normal nails. Ann Rheum Dis 2012;71:533-6.

16. McGonagle D, Ash Z, Dickie L, et al. The early phase of psoriatic arthritis. Ann Rheum Dis 2011; 70(Suppl 1):i71-6.

17. McGonagle D, McDermott MF. A proposed classification of the immunological diseases. PLoS Med 2006; 3:e297.

18. Reich K. The concept of psoriasis as a systemic inflammation: implications for disease management. JEADV 2012;26(Suppl 2):3-11. 